\title{
Estimating soil salinity with different fractional vegetation cover using remote sensing
}

\author{
Junrui Zhang', Zhitao Zhang ${ }^{2}$, Junying Chen², Haiying Chen², Jiming Jin², Jia Han², \\ Xintao Wang ${ }^{2}$, Zhishuang Song ${ }^{2}$, and Guangfei Wei ${ }^{2}$ \\ ${ }^{1}$ Northwest A\&F University \\ ${ }^{2}$ Northwest Agriculture and Forestry University
}

May 5, 2020

\begin{abstract}
Soil salinization is a serious restrictive factor of sustainable agricultural development, and its monitoring accuracy is mainly influenced by such factors as mineral composition, organic matter, and Fractional Vegetation Cover (FVC). Previous research mostly focused on the first two factors and the study of FVC is scarce and unsystematic. In order to systematically explore the effect of FVC, we monitored the soil salinization with different vegetation coverage in Jiefangzha Irrigation District in Inner Mongolia using satellite remote sensing. From May to August 2018, we carried out field sampling at different depths $(0-20 \mathrm{~cm}, 0-40 \mathrm{~cm}, 0-60 \mathrm{~cm})$ in each month, and calculated FVC and spectral covariates using GF-1 satellite images in the corresponding sampling period. Based on the FVC division criteria of Inner Mongolia, we took the following steps: (1) setting up control treatment A (the full data with undivided FVC,TA) and experimental treatment B (bare land, TB), C (mid-low FVC, TC), D (mid FVC, TD) and E (high FVC, TE); (2) conducting the Best Subset Selection (BSS) for all spectral covariates at different depths of each treatment; and (3) constructing the Soil Salt Content (SSC) inversion models by Partial Least Square Regression (PLSR), Cubist, and Extreme Learning Machine (ELM). The results indicated that classifying FVC could improve the stability and predictive ability of the models. The results can provide references for soil salinization prevention and agricultural production in Jiefangzha Irrigation District and other areas with the same vegetation cover.
\end{abstract}

\section{Hosted file}

LDD-manuscript.docx available at https://authorea.com/users/300339/articles/430133-

estimating-soil-salinity-with-different-fractional-vegetation-cover-using-remote-sensing 


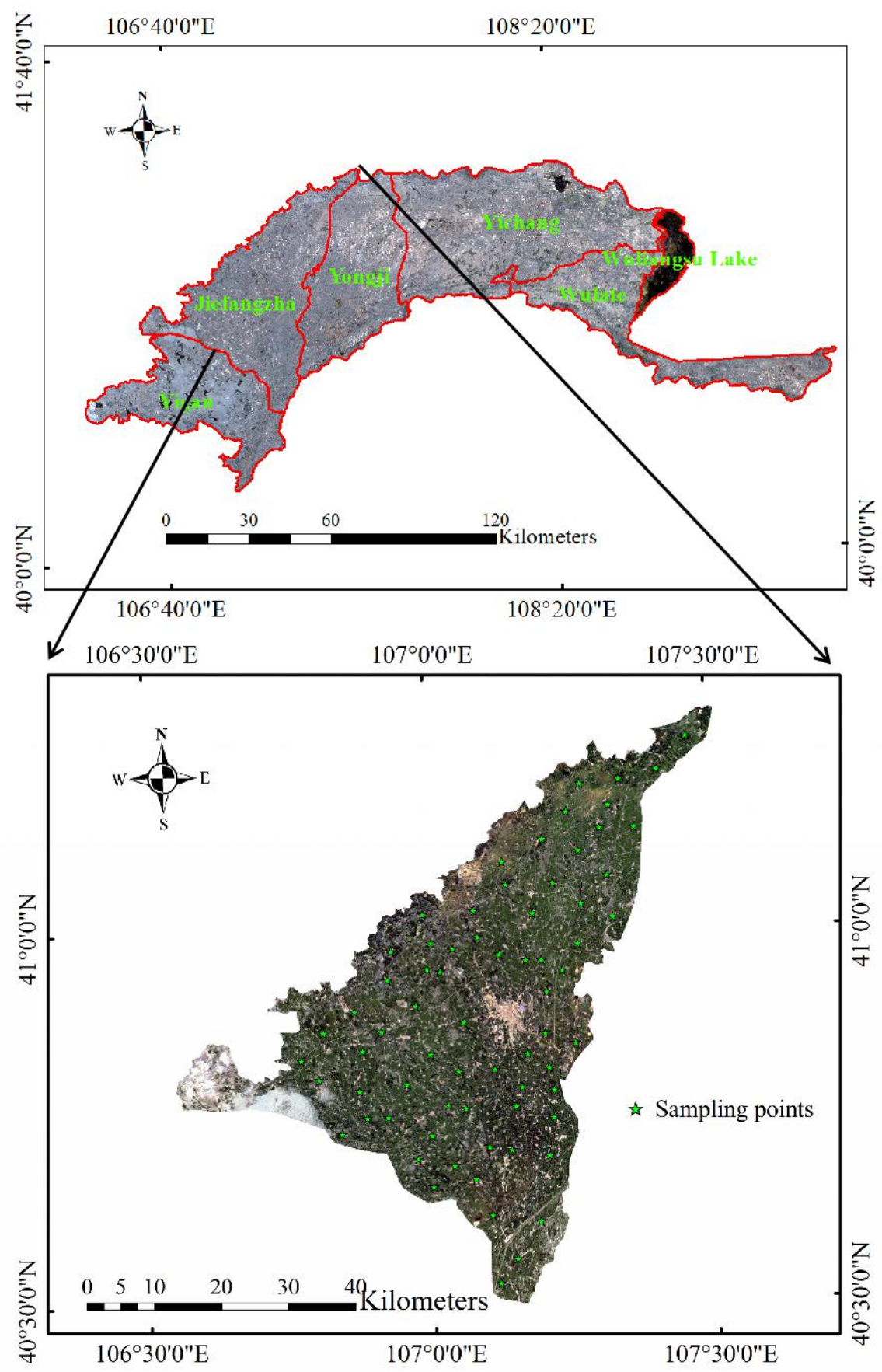



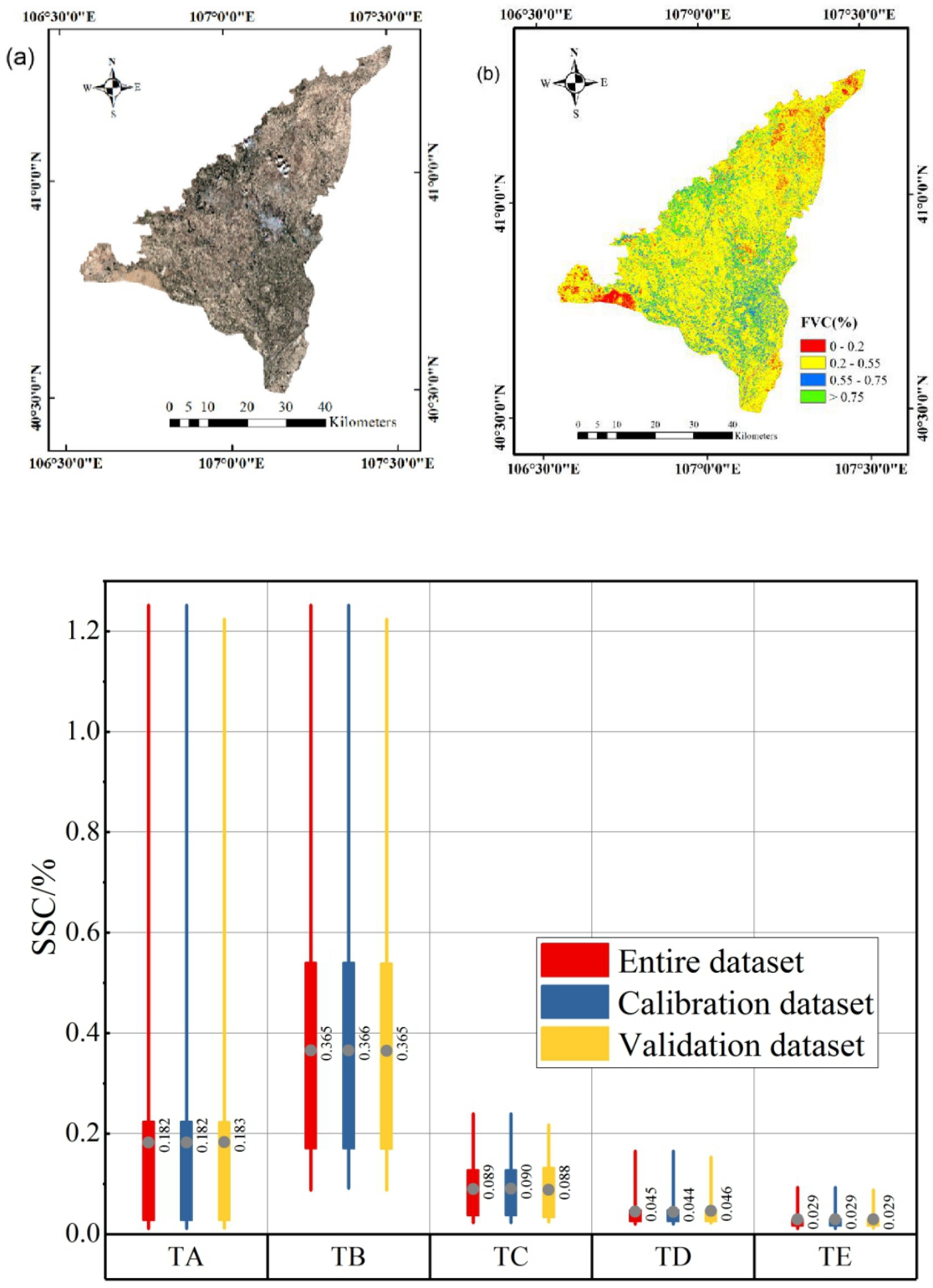


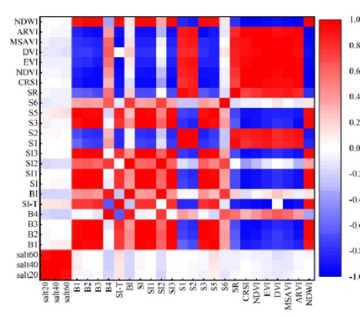

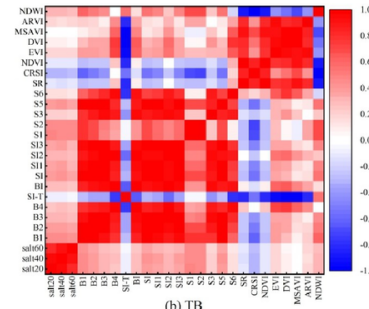
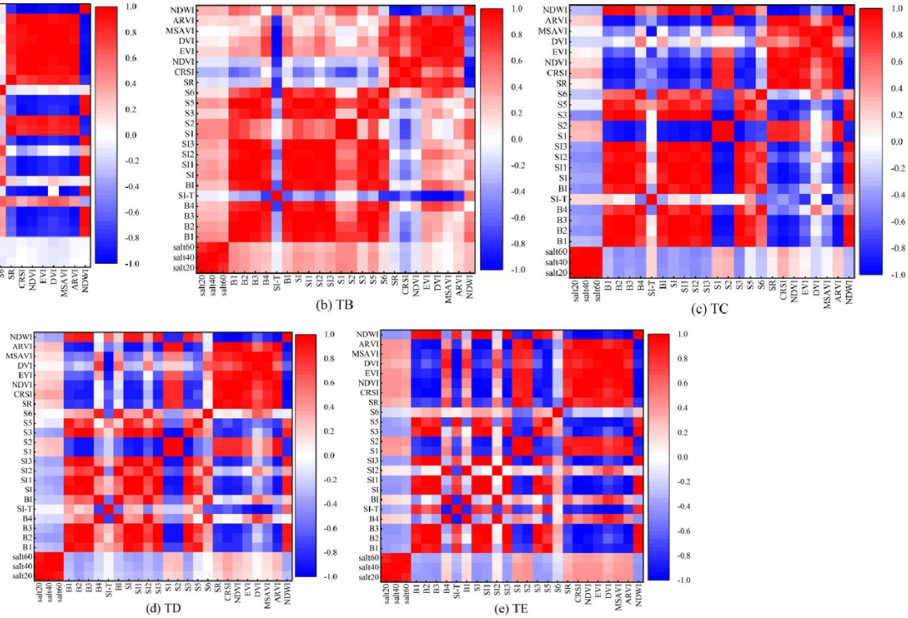

Calibration dataset
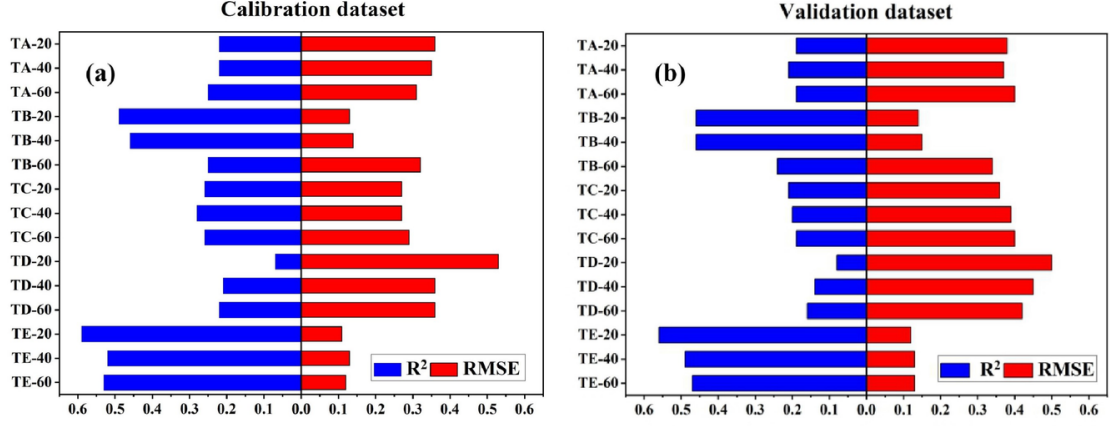

Calibration dataset

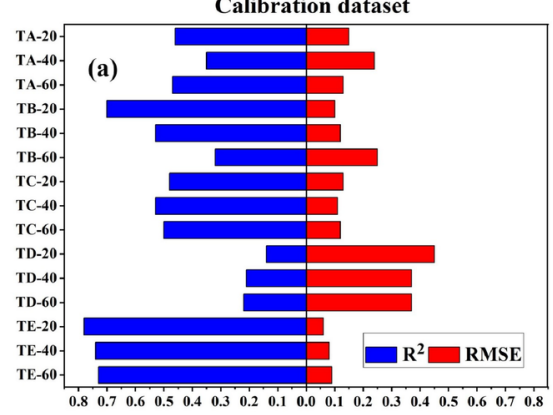

Validation dataset

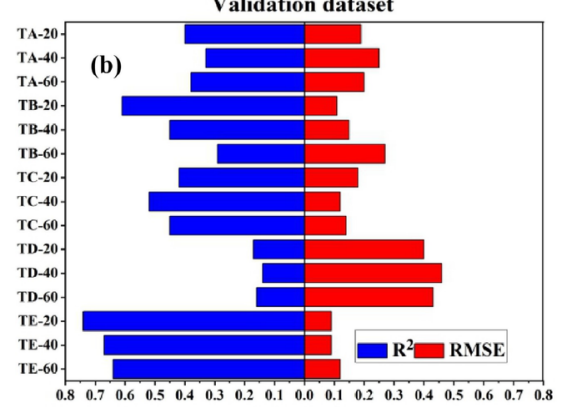



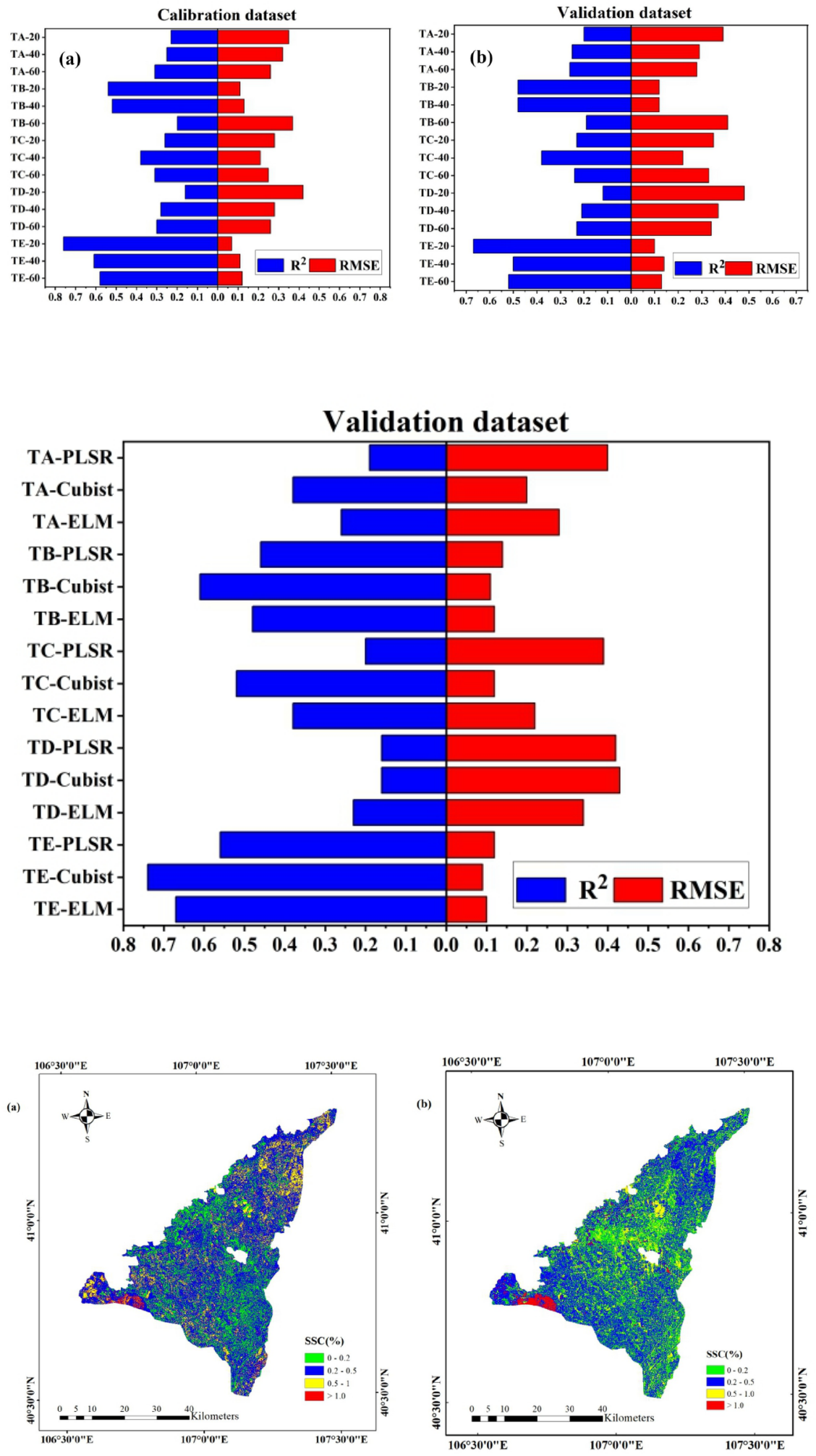

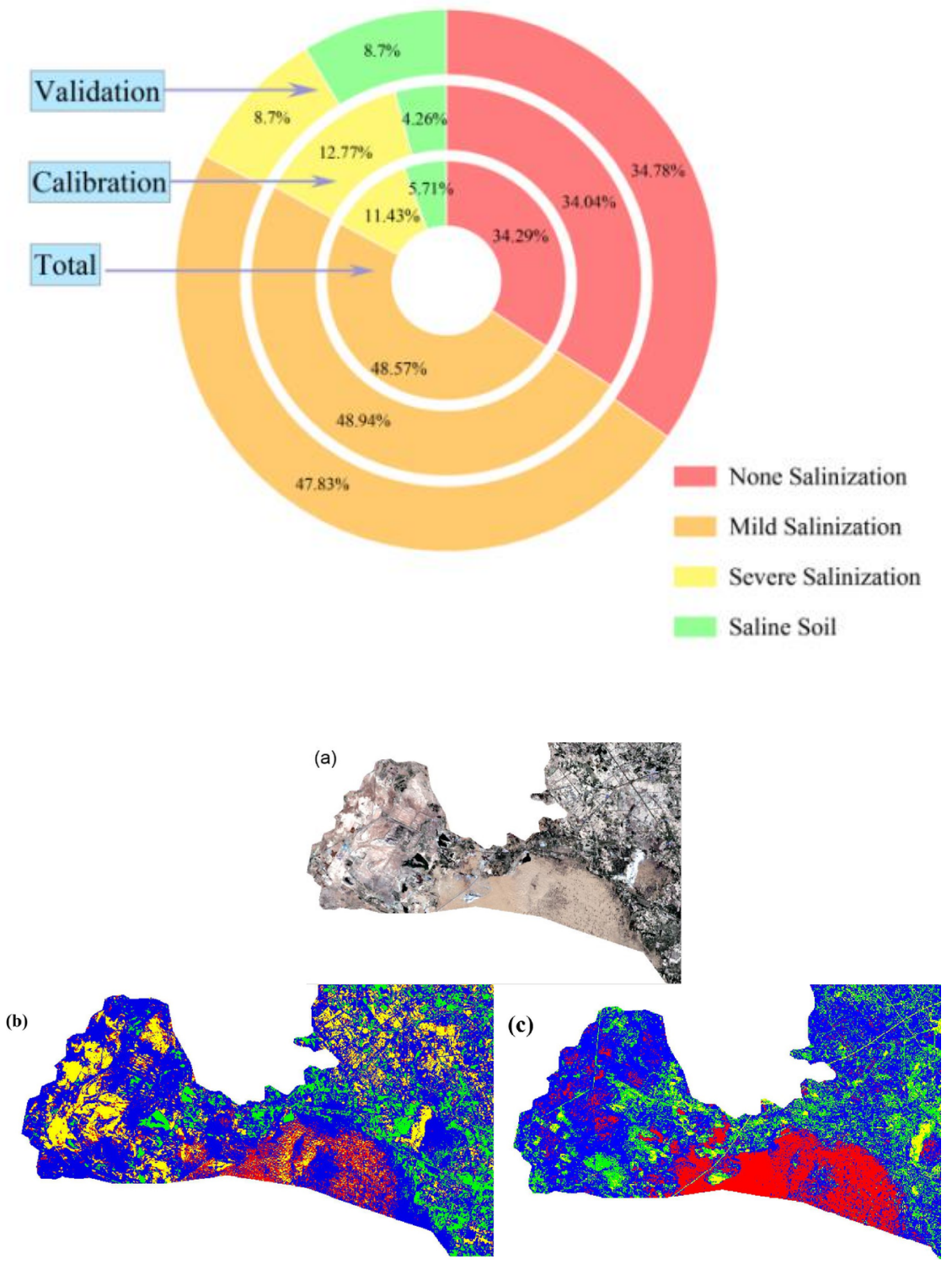